\title{
Pensions with heterogenous individuals and endogenous fertility $^{1}$
}

\author{
Helmuth Cremer, Firouz Gahvari and Pierre Pestieau \\ University of Toulouse (IDEI and GREMAQ) \\ 21, allée de Brienne \\ 31000 Toulouse - France \\ Department of Economics \\ University of Illinois at Urbana-Champaign \\ Champaign, IL 61820, USA \\ CREPP, University of Liège and CORE \\ 7, bd du Rectorat \\ 4000 Liège - Belgium
}

October 2004

\footnotetext{
${ }^{1}$ Paper to be presented at the CESifo Delta Conference (Munich, November 2004) "Optimal strategies for reforming PAYGO and funded pensions".
} 


\begin{abstract}
This paper studies the design of pension schemes in a society where fertility is endogenous and parents differ in their ability to raise children. In a world with perfect information, a pay-as-you-go social security system is characterized by equal pensions for all but different contributions which may or may not increase with the number of children. Additionally, fertility must be subsidized at the margin to correct for the externality that accompanies fertility. In a world of asymmetric information, incentiverelated distortions supplement the Pigouvian subsidy. These may either require an additional subsidy or an offsetting tax on fertility depending on whether the redistribution is towards people with more or less children. In the former case, pensions are decreasing in the number of children; in the latter case, they are increasing.
\end{abstract}




\section{Introduction}

The recent fertility decline in the West is often cited as a major impediment to the fiscal solvency of pay-as-you-go social security systems. At the same time, the pay-as-you-go feature of the social security systems has partly been blamed for causing the observed fertility decline. The reason for this latter linkage is that in such systems, the size of a person's pension benefits depends on everybody else's fertility decisions leading to a decentralized equilibrium outcome with "too few" children. It is thus not surprising that some economists have recently advocated a policy of linking pension benefits (or contributions) to individuals' fertility choices.

Such a policy raises a number of objections which one can group into "moral hazard" and "adverse selection" problems. The moral hazard problem arises when individuals do not have full control over fertility. The actual number of children in a family entails a random component and does not necessarily coincide with the number the parents initially intended to have. Making pension benefits to be independent of the number of children can then be viewed as a mechanism to insure parents against such random shocks. We have studied this problem in an earlier paper. ${ }^{1}$

The adverse selection problem, which is the subject of the current paper, arises when individuals are heterogenous. Specifically, assume, as is often the case, that parents differ in the ability to raise children (of a certain quality). Such individual characteristics are seldom publicly observable so that there is asymmetric information between parents and the policy makers. Under this circumstance, linking pension benefits to fertility penalizes high-cost families (the low-ability parents). This in turn may have an adverse redistributive impact. Put differently, it may not be possible to distinguish between those individuals who have a small number of children due to high costs, from those with low costs who try to free ride on the system. Consequently, the fertility-incentive effects of the pension system may have to be balanced against its redistributive impact.

This paper focuses on this potential trade-off between fertility incentives and redistribution. It studies the design of pension systems in a setting in which individuals differ

\footnotetext{
${ }^{1}$ Cremer et al. (2004a,b).
} 
in their cost of raising children (or alternatively in their preferences for their number of children). Specifically, we consider two mechanisms for financing pensions. The first relies on a storage technology which amounts to a fully funded system. The second is the pay-as-you-go formula wherein the rate of return depends on the rate of population growth. In this latter case, individuals' fertility decisions entail an externality that has to be taken into account in the design of the social security system. The paper's main message is that, even in the absence of moral hazard problems, the case for fertility related pension benefits is not as strong it may at first appear, and as it has been advocated in some recent work. ${ }^{2}$

\section{The model}

\subsection{The basics}

Consider a two-period overlapping generations model in the steady-state. Each generation consists of two types of individuals who differ in their "ability" to raise (productive) children. Each type is characterized by a specific cost of raising $n$ children

$$
k_{j}(n)=\theta_{j} n, \quad j=1,2,
$$

with $0<\theta_{2}<\theta_{1}$. Type 2 is thus the more able parent. All individuals are endowed with the same level of exogenous income, $y$, and have identical preferences over the number of children they will have, and present and future consumption.

Denote the proportion of type $j$ by $\pi_{j}$ and define average fertility as

$$
\bar{n}=\pi_{1} n_{1}+\pi_{2} n_{2}
$$

Introduce

$$
z_{j}=\theta_{j} n_{j}
$$

to denote a $j$-type parent's expenditure on children (excluding any subsidy he may receive, or any tax that he may have to pay, for this purpose). It will become clear below that whether $z_{2}>z_{1}$ or $z_{1}>z_{2}$, plays an important role in the type of solutions that emerge.

\footnotetext{
${ }^{2}$ Sinn (2004), Abio et al. (2004), Fenge and Meier (2004) Van Groezen et al. (2003).
} 
To keep the model simple, we assume that preferences over present and future consumption $\left(c_{j}, d_{j}\right)$ and the number of children, $n_{j}$, are represented by an additive utility function. The lifetime utility of an individual of type $j$ is written as

$$
U_{j}=u\left(c_{j}\right)+v\left(d_{j}\right)+h\left(n_{j}\right)
$$

where $u(\cdot), v(\cdot)$ and $h(\cdot)$ are strictly concave functions. There are two potential mechanism for financing second-period consumption: storage or a PAYGO pension plan. Under the storage technology, part of the initial endowment $y$ is invested in a fund yielding a fixed rate of return $r$. Under the PAYGO scheme, the government collects taxes from the current young and distributes the proceeds to the retired according to some rule to be designed. The rate of return of the PAYGO is $\bar{n}-1$. This corresponds to what Samuelson (1958) called the biological rate of interest.

\subsection{The Laissez-faire}

Absent any government intervention, each individual maximizes his utility (2) subject to his budget constraint

$$
y_{i}=c_{j}+n_{j} \theta_{j}+\frac{d_{j}}{1+r},
$$

where the pension (or savings) technology is the storage. Using the superscript $L$ for laissez-faire, the optimality conditions are

$$
\begin{aligned}
& (1+r) v^{\prime}\left(d_{j}^{L}\right)=u^{\prime}\left(c_{j}^{L}\right), \\
& h^{\prime}\left(n_{j}^{L}\right)=\theta_{j} u^{\prime}\left(c_{j}^{L}\right) .
\end{aligned}
$$

Given our assumptions on preferences, $c_{j}, d_{j}$ and $n_{j}$ are all normal goods. The normality of $n_{j}$ and the fact that $\theta_{2}<\theta_{1}$ then imply

$$
n_{2}^{L}>n_{1}^{L}
$$

The comparisons between the $c^{L}$ 's, the $d^{L}$ 's and the $z^{L}$ 's are ambiguous. However, given the specification for preferences, it must be the case that $c_{2}^{L}-c_{1}^{L}$ and $d_{2}^{L}-d_{1}^{L}$ are always of the same sign and opposite the sign of $z_{2}^{L}-z_{1}^{L}$. 
As an illustration, consider a logarithmic utility function,

$$
U_{j}=\alpha \ln c_{j}+\beta \ln d_{j}+\gamma \ln n_{j},
$$

where $\alpha+\beta+\gamma=1$. With this specification, the laissez-faire implies that consumption levels in both periods and expenditures on children are the same for all households:

$$
\begin{aligned}
c_{1}^{L} & =c_{2}^{L}=\alpha y, \\
d_{1}^{L} & =d_{2}^{L}=\beta(1+r) y, \\
z_{1}^{L} & =z_{2}^{L}=\gamma y .
\end{aligned}
$$

With a CES utility function, the comparison would depends on the elasticity of substitution. When this elasticity is small, the demand for $n$ is price inelastic and

$$
z_{1}^{L}>z_{2}^{L} .
$$

In other words, in this special case, the less able family has less children but spends more on raising them than the more able family does. A large elasticity of substitution yields the opposite result. Between these cases lies the Cobb-Douglas (logarithmic) specification with a unitary elasticity of substitution and constant budget shares.

\section{The utilitarian first-best}

Assume that the social planner controls all relevant variables in the economy and has perfect information regarding every individual's ability to raise children. The planner determines which technology, storage or PAYGO, is used to finance old-age consumption and it sets $c_{j}, d_{j}, n_{j}$ accordingly. We study the utilitarian solution which maximizes the sum of lifetime utility

$$
W=\sum_{j} \pi_{j} U_{j},
$$

subject to two alternative resource constraints:

$$
\begin{aligned}
\sum_{j} \pi_{j}\left(y_{j}-c_{j}-n_{j} \theta_{j}-\frac{d_{j}}{1+r}\right) & =0 \\
\sum_{j} \pi_{j}\left(y_{1}-c_{j}-n_{j} \theta_{j}-\frac{d_{j}}{\bar{n}}\right) & =0 .
\end{aligned}
$$




\subsection{Storage}

The storage problem can be expressed by the following Lagrangian expression

$$
\mathcal{L}_{F S}=\sum_{j} \pi_{j}\left[u\left(c_{j}\right)+v\left(d_{j}\right)+h\left(n_{j}\right)+\mu\left(y-c_{j}-\theta_{j} n_{j}-\frac{d_{j}}{1+r}\right)\right]
$$

where FS stands for "first-best under storage" and $\mu$ is the Lagrange multiplier associated with the economy's resource constraint. The first order conditions yield

$$
\begin{aligned}
& u^{\prime}\left(c_{1}^{F S}\right)=u^{\prime}\left(c_{2}^{F S}\right)=\mu, \\
& v^{\prime}\left(d_{1}^{F S}\right)=v^{\prime}\left(d_{2}^{F S}\right)=\frac{\mu}{1+r}, \\
& h^{\prime}\left(n_{j}^{F S}\right)=\mu \theta_{j} .
\end{aligned}
$$

The first two equations imply $c_{1}^{F S}=c_{2}^{F S}=c^{F S}, d_{1}^{F S}=d_{2}^{F S}=d^{F S}$, and the third equation $n_{2}^{F S}>n_{1}^{F S}$.

Decentralization of the first-best solution is simple. It requires first-period lump-sum tax and transfers between the two types while allowing them to save for their retirement voluntarily. Whether a type $j(j=1,2)$ person receives a transfer or will have to pay a tax depends on whether he spends more or less on child raising than a person of the other type. Specifically, if type 1 persons spend more than type 2, they should each receive a compensatory lump-sum transfer; if they spend less, they should pay a tax. Alternatively, decentralization can be achieved through a fully funded pension system where everyone receives the same pension but different types pay different contributions. Thus type $j$ persons each pay $y-c_{j}^{F S}-\theta_{j} z_{j}^{F S}$ ) when they work, and receive $d^{F S}$ when they retire. ${ }^{3}$ Observe that contributions may depend on family size either positively or negatively. If $z_{1}^{F S}>z_{2}^{F S}$, more able type 2 (who have a greater number of children) pay more in contributions. ${ }^{4}$ If $z_{1}^{F S}<z_{2}^{F S}$, the opposite holds and contributions decrease with family size.

\subsubsection{First best versus laissez faire under storage}

One can easily check that laissez faire and first-best solutions coincide if $z_{1}^{L}=z_{2}^{L}$. Moreover, it is also the case that if $z_{1}^{L}>z_{2}^{L}$, the first-best solution will be characterized

\footnotetext{
${ }^{3}$ The only variable left to choose by households is then the number of children.

${ }^{4}$ Recall that consumption levels are equalized in the first best.
} 
by $z_{1}^{F B}>z_{2}^{F B}$. Similarly, $z_{1}^{L}<z_{2}^{L} \Rightarrow z_{1}^{F B}<z_{2}^{F B}$. To see these, assume $z_{1}^{L}>z_{2}^{L}$. Under this circumstance, we have $c_{1}^{L}<c_{2}^{L}$ and $d_{1}^{L}<d_{2}^{L}$. To attain first-best, which requires the equality for consumption levels, one must then redistribute from type 2 to type 1 . With $n_{j}$ being a normal good, such a redistribution implies that $n_{1}^{F S}>n_{1}^{L}$ and $n_{2}^{F S}<n_{2}^{L}$. Consequently, $z_{1}^{F S}=\theta_{1} n_{1}^{F S}>\theta_{1} n_{1}^{L}>z_{1}^{L}$, and $z_{2}^{F S}=\theta_{2} n_{2}^{F S}<\theta_{2} n_{2}^{L}<z_{2}^{L}$. These inequalities then imply that $z_{1}^{F S}>z_{1}^{L}>z_{2}^{L}>z_{2}^{F S}$. A similar argument shows that if $z_{1}^{L}<z_{2}^{L}$, the corresponding first-best solution will be characterized by $z_{1}^{F B}<z_{2}^{F B}$.

Finally, observe that with the logarithmic utility (3), the first-best and the laissezfaire solutions coincide as $z_{1}^{L}=z_{2}^{L}$. In this case, pension contributions are the same for all individuals.

\subsection{Pay-as-you-go}

With PAYGO, the maximization of utilitarian welfare can be expressed by the following Lagrangian

$$
\mathcal{L}_{F P}=\sum_{j} \pi_{j}\left[n\left(c_{j}\right)+v\left(d_{j}\right)+h\left(n_{j}\right)+\mu\left(y-c_{j}-\theta_{j} n_{j}-\frac{d_{j}}{\bar{n}}\right)\right]
$$

where FP stands for "first-best with PAYGO". The optimality conditions are given by:

$$
\begin{aligned}
& u^{\prime}\left(c_{1}^{F P}\right)=u^{\prime}\left(c_{2}^{F P}\right)=\mu, \\
& v^{\prime}\left(d_{1}^{F P}\right)=v^{\prime}\left(d_{2}^{F P}\right)=\frac{\mu}{\bar{n}}, \\
& h^{\prime}\left(n_{j}^{F P}\right)=\mu\left(\theta_{j}-\frac{\pi_{1} d_{1}^{F P}+\pi_{2} d_{2}^{F P}}{\bar{n}^{2}}\right) .
\end{aligned}
$$

Equations (7) and (8) are standard; they imply $c_{1}^{F P}=c_{2}^{F P}=c^{F P}$ and $d_{1}^{F P}=d_{2}^{F P}=$ $d^{F P}$. Equation (9) has two interesting implications. The first is that $n_{1}^{F P}<n_{2}^{F P}$. That is, as with storage, the more productive individuals should have more children. Secondly, the equation shows that the existence of the PAYGO system affects the tradeoff between $c$ and $n$. To make this more explicit, one can rewrite (9) as

$$
\frac{h^{\prime}\left(n_{j}^{F P}\right)}{u^{\prime}\left(c_{j}^{F P}\right)}=\left(\theta_{j}-\frac{d^{F P}}{\bar{n}^{2}}\right)
$$


where $\bar{n}$ is also evaluated at its first-best value under PAYGO. The right-hand side of this expression represents the net marginal cost of $n$, accounting for the "externality" term $d^{F P} / \bar{n}^{2}$ which reflects the impact of one's fertility on the rate of return of the PAYGO system. ${ }^{5}$

To decentralize this solution, a Pigouvian subsidy at the rate of $s=d^{F P} / \bar{n}^{2}$ must supplement the pension system. Thus, with PAYGO, expenditures on children are subsidized at the margin. This was not the case under storage. The marginal subsidy implies that, under PAYGO, type 2 individuals who have a higher number of children will always receive a larger Pigouvian subsidy. ${ }^{6}$ Nevertheless, this does not imply that they are necessarily the beneficiaries of the pension system. The direction of net transfers between the types depends, once again, on the expenditures on children. If $z_{1}^{F P}>z_{2}^{F P}$, there will be a net transfer from low-cost households (with many children) to high-cost households (with fewer children); the opposite is true if $z_{1}^{F P}<z_{2}^{F P}$.

While individuals of different types receive the same pensions, $d^{F P}$, they will generally pay different contributions. Let $T_{j}$ denote the $j$-type's contribution. It then follows from the individuals' budget constraints that

$$
T_{1}-T_{2}=\left(\theta_{2}-\frac{d^{F P}}{\bar{n}^{2}}\right) n_{2}-\left(\theta_{1}-\frac{d^{F P}}{\bar{n}^{2}}\right) n_{1} .
$$

Observe that $T_{j}(j=1,2)$ will be greater than $T_{k}(k \neq j)$ if and only if the $j$-type's expenditure on children net of subsidies received is smaller than $k$-type's. Moreover, from the government's budget constraint,

$$
\sum_{j} \pi_{j} T_{j}-\frac{d^{F P}}{\bar{n}^{2}} \sum_{j} \pi_{j} n_{j}=\frac{d^{F P}}{\bar{n}} .
$$

\footnotetext{
${ }^{5}$ To be more precise, we have

$$
\frac{\partial \sum_{k} \pi_{k} d_{k}^{F P} / \bar{n}}{\partial n_{j}}=-\pi_{j} \frac{d^{F P}}{\bar{n}^{2}}, \quad j=1,2,
$$
}

with

$$
\begin{aligned}
\frac{\left.\partial \sum_{k} \pi_{k} h_{(} n_{k}\right)}{\partial n_{j}} & =\pi_{j} h^{\prime}\left(n_{j}\right) \\
\frac{\left.\partial \sum_{k} \pi_{k} u_{(} c_{k}\right)}{\partial c_{j}} & =\pi_{j} u^{\prime}\left(c_{j}\right) .
\end{aligned}
$$

\footnotetext{
${ }^{6}$ This argument assumes a linear Pigouvian subsidy scheme.
} 
Solving these two equations yields

$$
\begin{aligned}
& T_{1}=\frac{d^{F P}}{\bar{n}}+n_{1} \frac{d^{F P}}{\bar{n}^{2}}-\pi_{2}\left(z_{1}-z_{2}\right), \\
& T_{2}=\frac{d^{F P}}{\bar{n}}+n_{2} \frac{d^{F P}}{\bar{n}^{2}}-\pi_{1}\left(z_{2}-z_{1}\right) .
\end{aligned}
$$

Note that even in the Cobb-Douglas case, the contributions will be different. In this case, with $z_{1}=z_{2}$, we have

$$
\begin{aligned}
& T_{1}=\frac{d^{F P}}{\bar{n}}+n_{1} \frac{d^{F P}}{\bar{n}^{2}}, \\
& T_{2}=\frac{d^{F P}}{\bar{n}}+n_{2} \frac{d^{F P}}{\bar{n}^{2}} .
\end{aligned}
$$

\subsection{Storage versus PAYGO}

The choice between storage and PAYGO depends on the respective levels of welfare achieved, $W^{F S}$ and $W^{F P}$. This in turn will depend on the relationship between $1+r$, $\bar{n}^{F P}$, and $\bar{n}^{F S}$. However, the comparison is more complicated than in the standard Samuelsonian world. Specifically, if $1+r>\bar{n}^{F P}$, storage dominates PAYGO. Under this circumstance,

$$
\sum_{j} \pi_{j}\left(y-c_{j}^{F P}-\theta_{j} n_{j}^{F P}-\frac{d_{j}^{F P}}{1+r}\right)>\sum_{j} \pi_{j}\left(y-c_{j}^{F P}-\theta_{j} n_{j}^{F P}-\frac{d_{j}^{F P}}{\bar{n}^{F P}}\right)=0,
$$

so that the optimal allocation under PAYGO is also feasible under storage with a nonbinding resource constraint. Similarly, if $1+r=\bar{n}^{F P}$, storage continues to dominate PAYGO. In this case, the return of the two systems is the same, but we know from the results presented above that the allocations differ. Moreover, whereas $r$ is available on an exogenous basis, the return of PAYGO is endogenous and imposes restriction on the choice of $n$. Consequently, welfare must be strictly larger under storage.

The comparison is more involved if $1+r<\bar{n}^{F P} .7$ Specifically, if $1+r<\bar{n}^{F S}$,

$$
\sum_{j} \pi_{j}\left(y-c_{j}^{F S}-\theta_{j} n_{j}^{F S}-\frac{d_{j}^{F S}}{\bar{n}^{F S}}\right)>\sum_{j} \pi_{j}\left(y-c_{j}^{F S}-\theta_{j} n_{j}^{F S}-\frac{d_{j}^{F S}}{1+r}\right)=0,
$$

\footnotetext{
${ }^{7}$ In this case

$$
0=\sum_{j} \pi_{j}\left(y-c_{j}^{F S}-\theta_{j} n_{j}^{F S}-\frac{d_{j}^{F S}}{1+r}\right)<\sum_{j} \pi_{j}\left(y-c_{j}^{F S}-\theta_{j} n_{j}^{F S}-\frac{d_{j}^{F S}}{\bar{n}^{F P}}\right) .
$$
}

But $\bar{n}^{F P} \neq \bar{n}^{F S}=\pi_{1} n_{1}^{F S}+\pi_{2} n_{2}^{F S}$. 


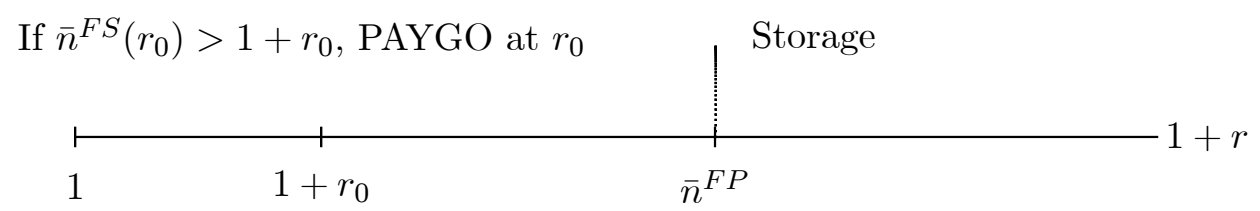

Figure 1: The choice between storage and PAYGO.

and the optimal allocation under storage is feasible under PAYGO with a non-binding resource constraint. Consequently, PAYGO dominates a storage technology that offers $r$ if $1+r<\bar{n}^{F S}(r)$. The comparison remains ambiguous if $\bar{n}^{F S}<1+r<\bar{n}^{F P}$. This is depicted in Figure 1. To the right of $\bar{n}^{F P}$, storage always dominates PAYGO. To the left of $\bar{n}^{F P}$, if, given any $r$, the resulting $\bar{n}^{F S}$ (which is a function of $r$ ) is greater than $1+r$, PAYGO dominates. Otherwise, the outcome is not clearcut. ${ }^{8}$

Finally, observe that the comparison between $n_{j}^{F S}$ and $n_{j}^{F P}$ is in general ambiguous. That $n_{j}$ will be subsidized under PAYGO but not under storage implies that, ceteris paribus, $n_{j}$ will be higher under PAYGO. However, the income effect may reverse this. To the left of $\bar{n}^{F P}$, the "real" purchasing power of resources is higher under PAYGO as compared to storage. This implies that $n_{j}^{F P}>n_{j}^{F S}$. To the right of $\bar{n}^{F P}$, the opposite is the case and the income effect leads one to choose a smaller value for $n_{j}$ under PAYGO. If $r$ is very large, income effect may dominate the substitution effect and we will have $n_{j}^{F P}<n_{j}^{F S}$.

\section{Second-best solution}

The first-best characterization rests on the assumption that the government observes $\theta_{j}$ and can use all instruments. If types are not publicly observable, one has to resort to a

\footnotetext{
${ }^{8}$ One can easily show that the sign of $d n_{j}^{F S}(r) / d r$ follows the sign of $d^{F S} /(1+r)+v^{\prime}\left(d^{F S}\right) / v^{\prime \prime}\left(d^{F S}\right)$, where $d^{F S}$ is a function of $r$. This can take positive as well as negative values and is not monotonic in $r$. Consequently, one cannot divide the interval to the left of $\bar{n}^{F P}$ into distinct subintervals over which PAYGO or storage dominate.
} 
tax-transfer policy which induces type revelation and leads to the appropriate fertility rate. Thus assume that $\theta_{j}$ is not observable but the number of children, $n_{j}$, is. The unobservability of types requires that $z_{j}=n_{j} \theta_{j}$ and $c_{j}$ not to be observable either. Otherwise, one could infer the value of $\theta_{j}$. However, the second-period consumption level, $d_{j}$, can be observable - an assumption that we maintain throughout this section.

To write the second-best problem in terms of observable variables, one must replace $c_{j}$ by $T_{j}$ (the first-period tax levied on the $j$-type). We can then determine the utilitarian allocation subject to the appropriate self-selection constraints. The solution can be decentralized through non-linear functions $T(n)$ and $d(n)$ which specify contributions and pensions as functions of the family size. Thus the $j$-type household chooses $n_{j}$ to maximize $u\left(y-T\left(n_{j}\right)-n_{j} \theta_{j}\right)+v\left(d\left(n_{j}\right)\right)+h\left(n_{j}\right)$. This yields the following first-order condition

$$
-u^{\prime}\left(y-T_{j}-n_{j} \theta_{j}\right)\left(\theta_{j}+T_{j}^{\prime}\right)+h^{\prime}\left(n_{j}\right)+v^{\prime}\left(d_{j}\right) d_{j}^{\prime}=0
$$

or

$$
T_{j}^{\prime}-d_{j}^{\prime} \frac{v^{\prime}\left(d_{j}\right)}{u^{\prime}\left(c_{j}\right)}=-\theta_{j}+\frac{h^{\prime}\left(n_{j}\right)}{u^{\prime}\left(c_{j}\right)}
$$

where $T_{j}^{\prime}=T^{\prime}\left(n_{j}\right)$ and $d_{j}^{\prime}=d^{\prime}\left(n_{j}\right)$. The left-hand-side of this expression specifies the net marginal tax on $n$ for household $j$. It has two components: a first-period tax, $T_{j}^{\prime}$, combined with a second-period transfer, $d_{j}^{\prime}$, weighted by the intertemporal marginal rate of substitution, $v^{\prime}\left(d_{j}\right) / u^{\prime}\left(c_{j}\right)$. In the discussion below, it is more convenient to speak of a subsidy rather than a tax; we thus define the marginal subsidy rate on $n_{j}$ as

$$
s_{j} \equiv-T_{j}^{\prime}+d_{j}^{\prime} \frac{v^{\prime}\left(d_{j}\right)}{u^{\prime}\left(c_{j}\right)},
$$

where, at the optimum, $s_{j}$ is set equal to $\theta_{j}-h^{\prime}\left(n_{j}\right) / u^{\prime}\left(c_{j}\right)$.

\subsection{Storage}

Let $c_{j k}$ and $U_{j k}(j \neq k=1,2)$ denote the consumption and the utility of a $j$-type who mimics a $k$-type. We have

$$
\begin{aligned}
c_{j k} & =y-\theta_{j} n_{k}-T_{k} \\
U_{j k} & =u\left(c_{j k}\right)+v\left(d_{k}\right)+h\left(n_{k}\right) .
\end{aligned}
$$


The optimal utilitarain allocation is obtained by maximizing the sum of individual utilities, subject to the resource constraint and the two potential self-selection constraints. The Lagrangian expression associated with this problem is given by

$$
\mathcal{L}=\sum_{j} \pi_{j}\left[U_{j}+\mu\left(T_{j}-\frac{d_{j}}{1+r}\right)\right]+\lambda_{2}\left(U_{2}-U_{21}\right)+\lambda_{1}\left(U_{1}-U_{12}\right) .
$$

The first-order conditions are:

$$
\begin{aligned}
\frac{\partial \mathcal{L}}{\partial T_{1}} & =-\left(\pi_{1}+\lambda_{1}\right) u^{\prime}\left(c_{1}\right)+\pi_{1} \mu+\lambda_{2} u^{\prime}\left(c_{21}\right)=0, \\
\frac{\partial \mathcal{L}}{\partial T_{2}} & =-\left(\pi_{2}+\lambda_{2}\right) u^{\prime}\left(c_{2}\right)+\pi_{2} \mu+\lambda_{1} u^{\prime}\left(c_{12}\right)=0 \\
\frac{\partial \mathcal{L}}{\partial d_{1}} & =\left(\pi_{1}+\lambda_{1}-\lambda_{2}\right) v^{\prime}\left(d_{1}\right)-\frac{\pi_{1} \mu}{(1+r)}=0, \\
\frac{\partial \mathcal{L}}{\partial d_{2}} & =\left(\pi_{2}+\lambda_{2}-\lambda_{1}\right) v^{\prime}\left(d_{2}\right)-\frac{\pi_{2} \mu}{(1+r)}=0, \\
\frac{\partial \mathcal{L}}{\partial n_{1}} & =-\left(\pi_{1}+\lambda_{1}\right)\left[u^{\prime}\left(c_{1}\right) \theta_{1}-h^{\prime}\left(n_{1}\right)\right]+\lambda_{2}\left[u^{\prime}\left(c_{21}\right) \theta_{2}-h^{\prime}\left(n_{1}\right)\right]=0, \\
\frac{\partial \mathcal{L}}{\partial n_{2}} & =-\left(\pi_{2}+\lambda_{2}\right)\left[u^{\prime}\left(c_{2}\right) \theta_{2}-h^{\prime}\left(n_{2}\right)\right]+\lambda_{1}\left[u^{\prime}\left(c_{12}\right) \theta_{1}-h^{\prime}\left(n_{2}\right)\right]=0 .
\end{aligned}
$$

Combining the first-order conditions (12) and (14) yields ${ }^{9}$

$$
v^{\prime}\left(d_{1}\right)=\frac{u^{\prime}\left(c_{1}\right)}{1+r}\left[\frac{\pi_{1}+\lambda_{1}-\lambda_{2} \frac{u^{\prime}\left(c_{21}\right)}{u^{\prime}\left(c_{1}\right)}}{\pi_{1}+\lambda_{1}-\lambda_{2}}\right] \geq \frac{u^{\prime}\left(c_{1}\right)}{1+r} .
$$

Similarly, (13) and (15) lead to

$$
v^{\prime}\left(d_{2}\right)=\frac{u^{\prime}\left(c_{2}\right)}{1+r}\left[\frac{\pi_{2}+\lambda_{2}-\lambda_{1} \frac{u^{\prime}\left(c_{12}\right)}{u^{\prime}\left(c_{2}\right)}}{\pi_{2}+\lambda_{2}-\lambda_{1}}\right] \leq \frac{u^{\prime}\left(c_{2}\right)}{1+r} .
$$

\footnotetext{
${ }^{9}$ It follows from (14) that $\pi_{1}+\lambda_{1}-\lambda_{2}>0$

and from (15) that$$
\pi_{2}+\lambda_{2}-\lambda_{1}>0
$$

Moreover, with $c_{j}=y-\theta_{j} n_{j}-T_{j}$,

$$
\begin{aligned}
& c_{21}-c_{1}=\left(\theta_{1}-\theta_{2}\right) n_{1}>0, \\
& c_{12}-c_{2}=\left(\theta_{2}-\theta_{1}\right) n_{2}<0 .
\end{aligned}
$$
}


Finally, combining (14) and (15) results in

$$
\frac{v^{\prime}\left(d_{2}\right)}{v^{\prime}\left(d_{1}\right)}=\frac{\pi_{2} \pi_{1}+\pi_{2}\left(\lambda_{1}-\lambda_{2}\right)}{\pi_{2} \pi_{1}+\pi_{1}\left(\lambda_{2}-\lambda_{1}\right)}
$$

Next, we distinguish between three regimes: $\lambda_{1}=\lambda_{2}=0, \lambda_{2}>0, \lambda_{1}=0$ and $\lambda_{2}=0, \lambda_{1}>0$.

Regime 1. $\lambda_{2}=\lambda_{1}=0$ This solution corresponds to the first-best where none of the self-selection constraints binds. It will necessarily hold if at the laissez-faire solution $z_{1}^{L}=z_{2}^{L}$ so that there is no need for redistribution. With the logarithmic utilities (3), we have precisely this case. More generally, this occurs whenever the first-best allocation satisfies the self-selection constraint. In turn, this arises when laissez-faire levels of expenditure on children are "not too different".

Regime 2. $\lambda_{2}>0$ and $\lambda_{1}=0$ In this regime, the prevailing self-selection constraint is that of type 2. Put differently, type 1 benefits from redistribution and type 2 is tempted to mimic him by having less children (i.e., by choosing $n_{1}$ rather than $n_{2}$ ). To discourage type 2 from mimicking type 1 , the social planner sets $d_{2}>d_{1}$. This follows immediately from (20). Consequently, in this regime, pensions increase with the family size. The comparison between $c_{1}$ and $c_{2}$, however, is ambiguous.

Turning to $n_{j}$ 's, we have from (17)

$$
\theta_{2}-\frac{h^{\prime}\left(n_{2}\right)}{u^{\prime}\left(c_{2}\right)}=0
$$

which implies there is no distortion in the choice of $n_{2}$. This is of course due to the fact that in this regime redistribution is from type 2 to type 1.

Next, from (16), one obtains

$$
\pi_{1}\left[u^{\prime}\left(c_{1}\right) \theta_{1}-h^{\prime}\left(n_{1}\right)\right]=\lambda_{2}\left[u^{\prime}\left(c_{21}\right) \theta_{2}-h^{\prime}\left(n_{1}\right)\right]
$$

implying

$$
\theta_{1}-\frac{h^{\prime}\left(n_{1}\right)}{u^{\prime}\left(c_{1}\right)}=\frac{\lambda_{2}}{\pi_{1}-\lambda_{2}} \frac{u^{\prime}\left(c_{21}\right) \theta_{2}-u^{\prime}\left(c_{1}\right) \theta_{1}}{u^{\prime}\left(c_{1}\right)}<0
$$


where $u^{\prime}\left(c_{21}\right) \theta_{2}-u^{\prime}\left(c_{1}\right) \theta_{1}<0$ because $c_{21}>c_{1}$ and $\theta_{2}<\theta_{1} \cdot{ }^{10}$ Consequently, there is a downward distortion in $n_{1}$ (as compared to the first-best tradeoff wherein $u^{\prime}\left(c_{1}\right) \theta_{1}-$ $\left.h^{\prime}\left(n_{1}\right)=0\right)$. In words, to discourage type 2 from mimicking type 1 , the social planner "suggests" a low value of $n_{1}$ that is not attractive to type 2 , given $h(\cdot)$ and $\theta_{2}$. This means, as a matter of implementation, there will be a tax on $n_{1}$.

Using (10)-(11), these results imply that $s_{2}=0$ while $s_{1}<0$. Consequently, marginal subsidy rates are non-positive for all households. While this in itself is not surprising (at least not under storage), it may appear to be at odds with the property that households with many children (type 2) receive higher pensions than households with fewer children. However, under closer scrutiny, it becomes clear that similar properties arise in standard optimal tax models where marginal and average tax rates do not always go hand in hand. ${ }^{11}$

Regime 3: $\lambda_{1}>0$ and $\lambda_{2}=0$ In this regime, type 2 benefits from redistribution and type 1 is tempted to mimic him by having more children. To counter this, the planner sets $d_{2}<d_{1}$ so that pensions now decrease with family size. This result is easily established from (20). Moreover, making use of (18) and (19), it is now also the case that $c_{2}<c_{1}$.

Turning to $n_{j}$ 's, (17) can now be rearranged to yield

$$
\theta_{2}-\frac{h^{\prime}\left(n_{2}\right)}{u^{\prime}\left(c_{2}\right)}=\frac{\lambda_{1}}{\pi_{2}-\lambda_{1}} \frac{u^{\prime}\left(c_{12}\right) \theta_{1}-u^{\prime}\left(c_{2}\right) \theta_{2}}{u^{\prime}\left(c_{2}\right)}>0,
$$

so that $n_{2}$ is distorted upward. In this regime, the binding incentive constraint is to prevent type 1 households from mimicking type 2 households by having more children. To relax this constraint, the social planner induces type 2 to have even more children (than they would in the laissez-faire).

In the case of type 1 parents, one may easily show from (16) that no distortion is to be imposed on $n_{1}$. This is not surprising as, in this regime, redistribution is away from type 1 and towards type 2. Using (10)-(11), these results imply that, in terms of

\footnotetext{
${ }^{10}$ With $\lambda_{1}=0$, from (14), $\pi_{1}-\lambda_{2}>0$.

${ }^{11}$ For example, in the "normal case" of Stiglitz's two-group model where the redistribution is from high- to low-ability persons, low-ability individuals face a positive marginal income tax rate while their average tax rate is negative.
} 
implementation, $s_{1}=0$ and $s_{2}>0$ so that marginal subsidy rates are non-negative for all types of households.

Which regime? We have already seen that if the two types spend equal amounts on raising children in the laissez faire, i.e. if $z_{1}^{L}=z_{2}^{L}$, the laissez faire and the optimal utilitarian solutions coincide and regime 1 necessarily prevails. We show in the Appendix that if $z_{1}^{L}<z_{2}^{L}$ the prevailing regime is either 1 or 2 , while if $z_{1}^{L}>z_{2}^{L}$, we will have regime 1 or 3 . Put differently, if the utilitarian solution calls for redistribution, it will be towards the parents who spend more on raising children. Thus the self-selection constraint that may constraint the extent of the redistribution will be that of the parent who spends the least on his children.

Recall that in the first best, there is full compensation for the differences in expenditures with the consumption levels being equalized across types. In a world of asymmetric information, this full equalization may or may not be achievable. When it is not, redistribution is limited by the binding incentive constraints of parents who lose from redistribution; namely, those who spend the least on raising their children. If these are the parents who have more children (type 2), then to make the alternative less appealing to them $n_{1}$ is distorted downward and $d_{1}$ is set less than $d_{2}$. On the other hand, if the losers are the parent with less children, their alternative is made less appealing by distorting $n_{2}$ upward and setting $d_{2}<d_{1}$.

\subsection{PAYGO}

Assume now that the government controls $d_{j}$ 's through pensions, setting them at levels such that there will be no private savings. One can then write the individual's budget constraint as

$$
c_{j}+\theta_{j} n_{j}+T^{j}=y
$$

and the resource constraint by

$$
\sum_{j=1}^{2} \pi_{j}\left(T_{j}-\frac{d_{j}}{\bar{n}}\right)=0
$$


As with the storage, let $c_{j k}$ and $U_{j k}(j \neq k=1,2)$ denote the consumption and the utility of a $j$-type who mimics a $k$-type. We have

$$
\begin{aligned}
& c_{j k}=y-\theta_{j} n_{k}-T_{k} \\
& U_{j k}=u\left(c_{j k}\right)+v\left(d_{k}\right)+h\left(n_{k}\right) .
\end{aligned}
$$

The second-best problem is then summarized by the Lagrangian

$$
\mathcal{L}=\sum_{j} \pi_{j}\left[U_{j}+\mu\left(T_{j}-\frac{d_{j}}{\bar{n}}\right)\right]+\lambda_{2}\left(U_{2}-U_{21}\right)+\lambda_{1}\left(U_{1}-U_{12}\right)
$$

where $\bar{n}$ is defined by (1).

The first-order conditions for this problem are

$$
\begin{aligned}
\frac{\partial \mathcal{L}}{\partial T_{1}}= & -\left(\pi_{1}+\lambda_{1}\right) u^{\prime}\left(c_{1}\right)+\pi_{1} \mu+\lambda_{2} u^{\prime}\left(c_{21}\right)=0, \\
\frac{\partial \mathcal{L}}{\partial T_{2}}= & -\left(\pi_{2}+\lambda_{2}\right) u^{\prime}\left(c_{2}\right)+\pi_{2} \mu+\lambda_{1} u^{\prime}\left(c_{12}\right)=0, \\
\frac{\partial \mathcal{L}}{\partial d_{1}}= & \left(\pi_{1}+\lambda_{1}-\lambda_{2}\right) v^{\prime}\left(d_{1}\right)-\frac{\pi_{1} \mu}{\bar{n}}=0, \\
\frac{\partial \mathcal{L}}{\partial d_{2}}= & \left(\pi_{2}+\lambda_{2}-\lambda_{1}\right) v^{\prime}\left(d_{2}\right)-\frac{\pi_{2} \mu}{\bar{n}}=0, \\
\frac{\partial \mathcal{L}}{\partial n_{1}}= & -\left(\pi_{1}+\lambda_{1}\right)\left[u^{\prime}\left(c_{1}\right) \theta_{1}-h^{\prime}\left(n_{1}\right)\right]+\pi_{1} \mu \frac{\pi_{1} d_{1}+\pi_{2} d_{2}}{\bar{n}^{2}} \\
& +\lambda_{2}\left[u^{\prime}\left(c_{21}\right) \theta_{2}-h^{\prime}\left(n_{1}\right)\right]=0, \\
\frac{\partial \mathcal{L}}{\partial n_{2}}= & -\left(\pi_{2}+\lambda_{2}\right)\left[u^{\prime}\left(c_{2}\right) \theta_{2}-h^{\prime}\left(n_{2}\right)\right]+\pi_{2} \mu \frac{\pi_{1} d_{1}+\pi_{2} d_{2}}{\bar{n}^{2}} \\
& +\lambda_{1}\left[u^{\prime}\left(c_{12}\right) \theta_{1}-h^{\prime}\left(n_{2}\right)\right]=0 .
\end{aligned}
$$

Observe that the first-order conditions with respect to $n_{j}$, i.e. equations (25) and (26), differ from their storage counterparts. On the other hand, expressions (21)-(24) are equivalent to $(12-(15)$ under the storage, except that $\bar{n}$ has replaced $(1+r)$. Similar manipulations of these equations then yield

$$
\begin{aligned}
v^{\prime}\left(d_{1}\right) & \geq \frac{1}{\bar{n}} u^{\prime}\left(c_{1}\right), \\
v^{\prime}\left(d_{2}\right) & \leq \frac{1}{\bar{n}} u^{\prime}\left(c_{2}\right),
\end{aligned}
$$

along with equation (20) which continues to hold. We again have three possible regimes. 
Regime 1: $\lambda_{2}=0$ and $\lambda_{1}=0$ The solution corresponds to the first best. Pensions are set equally $\left(d_{1}=d_{2}\right)$ and lump-sum taxes (contributions) are used to ensure $c_{1}=c_{2}$. Additionally, a Pigouvian subsidy is used to induce the optimal values of $n_{j}$ 's. In the Cobb-Douglas case, where $z_{1}^{L}=z_{2}^{L}$, there is no net redistribution between the two types. But with $n_{2}>n_{1}$ in the first best, type-2 receives more subsidy for raising children. Consequently, they will have to be taxed in the first period to ensure there will be no net transfers.

Regime 2: $\lambda_{2}>0$ and $\lambda_{1}=0$ Comparisons between the consumption and pension levels of the two types are exactly the same as in the storage. Specifically, it follows from (20) that $d_{2}>d_{1}$ and pensions increase with the family size. The comparison between $c_{1}$ and $c_{2}$ remains ambiguous.

Regarding $n_{j}$, for individuals of type 2 , we have from (26)

$$
-\left(\pi_{2}+\lambda_{2}\right)\left[u^{\prime}\left(c_{2}\right) \theta_{2}-h^{\prime}\left(n_{2}\right)\right]+\pi_{2} \mu \frac{\pi_{1} d_{1}+\pi_{2} d_{2}}{\bar{n}^{2}}=0
$$

Rearranging and using (22) gives

$$
\theta_{2}-\frac{h^{\prime}\left(n_{2}\right)}{u^{\prime}\left(c_{2}\right)}=\frac{\pi_{1} d_{1}+\pi_{2} d_{2}}{\bar{n}^{2}}
$$

so that

$$
s_{2}=\frac{\pi_{1} d_{1}+\pi_{2} d_{2}}{\bar{n}^{2}}
$$

In words, there is a "Pigouvian" marginal subsidy on $n$ for type 2 individuals. Observe that, as with storage, with the redistribution being away from type 2, incentive considerations require no distortion to be imposed on type 2 .

Turning to type-1 individuals, we have from (25),

$$
\pi_{1}\left[u^{\prime}\left(c_{1}\right) \theta_{1}-h^{\prime}\left(n_{1}\right)\right]=\pi_{1} \mu \frac{\pi_{1} d_{1}+\pi_{2} d_{2}}{\bar{n}^{2}}+\lambda_{2}\left[u^{\prime}\left(c_{21}\right) \theta_{2}-h^{\prime}\left(n_{1}\right)\right],
$$

so that

$$
\left(\pi_{1}-\lambda_{2}\right)\left[u^{\prime}\left(c_{1}\right) \theta_{1}-h^{\prime}\left(n_{1}\right)\right]=\pi_{1} \mu \frac{\pi_{1} d_{1}+\pi_{2} d_{2}}{\bar{n}^{2}}+\lambda_{2}\left[u^{\prime}\left(c_{21}\right) \theta_{2}-u^{\prime}\left(c_{1}\right) \theta_{1}\right],
$$


where $u^{\prime}\left(c_{21}\right) \theta_{2}-u^{\prime}\left(c_{1}\right) \theta_{1}<0$. Rearranging then yields

$$
\theta_{1}-\frac{h^{\prime}\left(n_{1}\right)}{u^{\prime}\left(c_{1}\right)}=\frac{\pi_{1} \mu}{\left(\pi_{1}-\lambda_{2}\right) u^{\prime}\left(c_{1}\right)} \frac{\pi_{1} d_{1}+\pi_{2} d_{2}}{\bar{n}^{2}}+\frac{\lambda_{2}}{\pi_{1}-\lambda_{2}} \frac{u^{\prime}\left(c_{21}\right) \theta_{2}-u^{\prime}\left(c_{1}\right) \theta_{1}}{u^{\prime}\left(c_{1}\right)} .
$$

The first term in the right-hand side of (29) is the Pigouvian subsidy (adjusted by the fact that the "marginal cost of public fund" is no longer equal to one). The second term is the distortion aimed at relaxing the binding incentive constraint of type 2 households (who are hurt by redistribution). As with the storage, this term is negative thus inducing a downward distortion on $n_{1}$. Consequently, the sign of $s_{1}$ is ambiguous. There is a conflict between externality (requiring a subsidy to induce a higher value for $n_{1}$ ) and incentive (requiring a tax to induce a lower value for $n_{1}$ ) terms. ${ }^{12}$

Regime 3: $\lambda_{1}>0$ and $\lambda_{2}=0$ The comparisons between consumption and pension levels are, once again, exactly the same as with storage. Specifically, it follows from (20) that $d_{2}<d_{1}$. Hence pensions decrease with family size, even though fertility entails a positive externality. Moreover, making use of (27) and (28) we obtain, as with storage, $c_{2}<c_{1}$.

Turning to $n_{j}$ 's, we again consider type-2 households first. One obtains, from (26),

$$
\left(\pi_{2}-\lambda_{1}\right)\left[u^{\prime}\left(c_{2}\right) \theta_{2}-h^{\prime}\left(n_{2}\right)\right]=\pi_{2} \mu \frac{\pi_{1} d_{1}+\pi_{2} d_{2}}{\bar{n}^{2}}+\lambda_{1}\left[u^{\prime}\left(c_{12}\right) \theta_{1}-u^{\prime}\left(c_{2}\right) \theta_{2}\right],
$$

where $u^{\prime}\left(c_{12}\right) \theta_{1}-u^{\prime}\left(c_{2}\right) \theta_{2}>0$. Rearranging yields

$$
\theta_{2}-\frac{h^{\prime}\left(n_{2}\right)}{u^{\prime}\left(c_{2}\right)}=\frac{\pi_{2} \mu}{\left(\pi_{2}-\lambda_{1}\right) u^{\prime}\left(c_{2}\right)} \frac{\pi_{1} d_{1}+\pi_{2} d_{2}}{\bar{n}^{2}}+\frac{\lambda_{1}}{\pi_{2}-\lambda_{1}} \frac{u^{\prime}\left(c_{12}\right) \theta_{1}-u^{\prime}\left(c_{2}\right) \theta_{2}}{u^{\prime}\left(c_{2}\right)} .
$$

In this case, externality and incentive terms are of the same sign and we necessarily have a marginal subsidy on $n_{2}$ (a negative marginal tax). This implies that $s_{2}>0$.

In the case of type- 1 parents, proceeding in the same manner as in regime 2 , one obtains from (25) that they should face a Pigouvian marginal subsidy. Specifically,

$$
s_{1}=\frac{\pi_{1} d_{1}+\pi_{2} d_{2}}{\bar{n}^{2}},
$$

with no distortions due to incentive considerations (recall that redistribution is away from type 1).

\footnotetext{
${ }^{12}$ Recall that $s$ is the marginal subsidy. A negative value thus means a positive marginal tax.
} 


\section{Conclusion}

This paper has shown that the design of a pension system depends mainly on two factors: whether the system is based on storage or is PAYGO, and the relative size of the expenditures on raising children incurred by the two types. The first factor is related to the inherent externality in a PAYGO social security system. Increasing the number of one's (productive) children bestows a positive externality on others by increasing the biological rate of return of the system. This is operative only in the PAYGO setting and its internalization requires a subsidy on having children. The second factor is related to the elasticity of substitution between consumption and fertility. In case of unitary elasticity (when the utility function is logarithmic) both types spend the same amount on raising children and there is no need for redistribution. When one type spends more, that type should be "compensated".

We have shown that if the redistribution is from type 1 (who have fewer children) to type 2 (who have more children), one must distort $n_{2}$ upward for incentive considerations. This will reinforce the externality correction (requiring a Pigouvian marginal subsidy) and the net effect requires a subsidy on $n_{2}$. On the other hand, if the redistribution is towards type 1 , one must distort $n_{1}$ downward. This requires a tax on $n_{1}$ and works in opposite direction to the marginal Pigouvian subsidy. The final outcome would then depend on the relative values of the distortion due to incentives and the Pigouvian term. In both cases, the person whom one redistributes away from, must face a Pigouvian subsidy only with no incentive-related distortion. These results suggests a distinction between net redistributive goals achieved by average taxation, and changing the behavior at the margin achieved by marginal taxation. 


\section{Appendix}

\section{A1 The prevailing regimes at the second-best optimum under storage}

We show that if $z_{1}^{L}>z_{2}^{L}$, the incentive constraint of type 1 individuals cannot be binding at the second-best optimum. Consequently, in this case, regime 2 cannot prevail and one has either regime 1 or regime 3 . Formally, we have

Lemma 1 If $z_{1}^{L}>z_{2}^{L}$, then at the second-best optimum under storage, one cannot have both $\lambda_{1}>0$ and $\lambda_{2}=0$.

Proof. The proof is by contradiction. Assume $\lambda_{1}>0$ and $\lambda_{2}=0$ so that the incentive constraint $U_{1} \geq U_{12}$ is binding. Using superscript $S S$ for the second-best optimum under storage, the binding incentive constraint implies

$$
u\left(c_{1}^{S S}\right)+v\left(d_{1}^{S S}\right)+h\left(n_{1}^{S S}\right)=u\left(c_{12}^{S S}\right)+v\left(d_{2}^{S S}\right)+h\left(n_{2}^{S S}\right)
$$

where

$$
\begin{aligned}
& c_{1}^{S S}=y-\theta_{1} n_{1}^{S S}-T_{1}^{S S}, \\
& c_{12}^{S S}=y-\theta_{1} n_{2}^{S S}-T_{2}^{S S} .
\end{aligned}
$$

Next define

$$
\begin{aligned}
\Psi_{i}(\Delta)= & \max _{c, d, n} u(c)+v(d)+h(n) \\
& \text { s.t. } c+\frac{d}{1+r}+\theta_{i} n=y+\Delta ;
\end{aligned}
$$

so that $\Psi_{i}(\Delta)$ is household $i$ 's $(i=1,2)$ maximal utility when facing the budget constraint (A3) and the (given) net transfer $\Delta$. With $\lambda_{1}>0$ and $\lambda_{2}=0$, first-order conditions (12), (14) and (16) imply that $\left(c_{1}^{S S}, d_{1}^{S S}, n_{1}^{S S}\right)$ solves problem (A2)-(A3) for household 1 given

$$
\Delta=\Delta_{1}^{S S}=\frac{d_{1}^{S S}}{1+r}-T_{1}^{S S}
$$


In words, allocation $\left(c_{1}^{S S}, d_{1}^{S S}, n_{1}^{S S}\right)$ yields the maximal utility for type 1 households given $\Delta=\Delta_{1}^{S S} \cdot{ }^{13}$ Clearly then, only if $\Delta>\Delta_{1}^{S S}$, it will be possible for type 1 to have same level of utility at another allocation. Consequently, to have (A1) satisfied, the $\Delta$ associated with $\left(c_{12}^{S S}, d_{2}^{S S}, n_{2}^{S S}\right), \Delta_{2}^{S S}$, must be greater than $\Delta_{1}^{S S}$. Making use of the resource constraint under storage, it follows that

$$
\Delta_{1}^{S S}<0<\Delta_{2}^{S S}=\frac{d_{2}^{S S}}{1+r}-T_{2}^{S S},
$$

i.e., there is a net transfer from type 1 to type 2 .

Finally define

$$
W\left(\Delta_{1}\right)=\pi_{1} \Psi_{1}\left(\Delta_{1}\right)+\pi_{2} \Psi_{2}\left(-\frac{\pi_{1} \Delta_{1}}{\pi_{2}}\right) .
$$

Thus $W($.$) represents the maximum utilitarian welfare, in the absence of the incentive$ constraints, as a function of the net transfers to type 1 households (with the transfers to type 2 being determined by the resource constraint). It thus follows from the definition of $W($.$) that$

$$
\sum_{i} \pi_{i} U_{i}^{S S} \leq W\left(\Delta_{1}^{S S}\right)
$$

Now differentiating (A4), using the envelope theorem, yields

$$
W^{\prime}\left(\Delta_{1}\right)=\pi_{1}\left(u^{\prime}\left(c_{1}\right)-u^{\prime}\left(c_{2}\right)\right)
$$

so that $W($.$) is increasing in \Delta_{1}$ if and only if $c_{1}<c_{2}$. But, with $z_{1}^{L}>z_{2}^{L}$, the normality of $c$ implies $c_{1}<c_{2}$ whenever $\Delta_{1}<0$. Consequently, $W($.$) is increasing in \Delta_{1}$ whenever $\Delta_{1}<0$. Hence $\Delta_{1}^{S S}<0$ together with (A5) yields

$$
\sum_{i} \pi_{i} U_{i}^{S S} \leq W\left(\Delta_{1}^{S S}\right)<W(0)
$$

Given that the laissez-faire solution corresponds to $\Delta_{1}=0$, the above inequality implies that welfare at the second best optimum is smaller than welfare at the laissez-faire solution. This is a contradiction because the laissez-faire is feasible in the second-best.

In exactly the same way, one can prove that

\footnotetext{
${ }^{13}$ Recall that we have the "no distortion at the top" property for type 1 here.
} 
Lemma 2 If $z_{1}^{L}<z_{2}^{L}$, then at the second-best optimum under storage, one cannot have both $\lambda_{1}=0$ and $\lambda_{2}>0$.

Thus, if $z_{1}^{L}<z_{2}^{L}$, regime 3 cannot prevail, and one has either regime 1 or regime 2 . 


\section{References}

[1] Abio, G., G. Mathieu and C. Patxot, (2004), On the optimality of PAYG pension systems in an endogenous fertility setting, Journal of Pension Finance.

[2] Bento, B., (1989), The old age security hypothesis and optimal population growth, Journal of Population Economics, 1, 285-301.

[3] Cigno, A., Luporini, A. and A. Pettini, (2003), Transfers to families with children as a principal agent problem, Journal of Public Economics, 87, 1165-1177.

[4] Cigno, A., (1991), The Economics of the Family, Clarendon Press, Oxford.

[5] Cremer, H., F. Gahvari and P. Pestieau, (2004a), Pensions with heterogeneous agents and endogenous fertility, mimeo.

[6] Cremer, H., F. Gahvari and P. Pestieau, (2004b), Pensions with endogenous and stochastic fertility, CORE Discussion Papers 2004/50.

[7] Cremer, H., F. Gahvari and P. Pestieau, (2003), Stochastic fertility, moral hazard, and the design of pay-as-you-go pension plans, paper presented at CESifo Venice Summer Institute.

[8] Fenge, R. and V. Meier, (2004), Pensions fertility incentives, Canadian Journal of Economics, (forthcoming).

[9] Samuelson, P.A., (1958), An exact consumption-loan model of interest with or without the social contrivance of money, Journal of Political Economy, 66, 467482.

[10] Sinn, H.W., (2004), The pay-as-you-go pension system as fertility insurance and an enforcement device, Journal of Public Economics, 88, 1335-1357.

[11] van Groezen, B., T. Leers and L. Meijdam, (2003), Social security and endogenous fertility: pensions and child allowances as Siamese twins, Journal of Public Economics, 87, 133-251. 